\title{
B16alphaGal Melanoma Vaccine
}

\author{
National Cancer Institute
}

\section{Source}

National Cancer Institute. B16alphaGal Melanoma Vaccine. NCI Thesaurus. Code C80046.

\begin{abstract}
A whole cell melanoma cancer vaccine with potential immunostimulating and antineoplastic activities. B16alphaGal melanoma vaccine contains three types of human melanoma cell lines that are genetically eng ineered to express the alpha(1,3)-galactosyl (alphaGal) epitope on cell surfaces. The agent stimulates a hyperacute rejection of whole melanoma cancer cells expressing alphaGal epitopes, initiated by opsonization by antialphaGal antibodies and followed by antibody-dependent cell-mediated cytotoxicity (ADCC) and cell lysis. This results in the stimulation of a broader cytotoxic T-lymphocyte response $(C T L)$ directed against tumor antigens on melanoma cells that do not express alphaGal. AlphaGal is not normally expressed in humans because alpha(1,3)galactosyltransferase (Alpha-GT), the enzyme that catalyzes the synthesis of alphaGal epitopes on glycoproteins and glycolipids, is not naturally present in humans and other primates.
\end{abstract}

